\title{
Extent of Professional Practice of Physical Therapists
}

\author{
NICOLYNE B. MONSANTO \\ ORCID No. 0000-0003-2566-8114 \\ nikoymonsanto@gmail.com
}

AR-AN J. NANOL

ORCID No. 0000-0003-0796-4083

kevin_7981@yahoo.com

DIONESIO A. PONGO, JR.

ORCID No. 0000-0002-4495-7176

ldcupongo@yahoo.com.ph

DENISE O. ORONG

ORCID No. 0000-0002-2518-7866

nonoyorong@gmail.com

Liceo de Cagayan University

Cagayan de Oro City, Philippines

\begin{abstract}
The clinical practice of physical therapy in the Philippines is moving towards full autonomy and direct access to patients. This development makes a physical therapist becomes a primary care contact and that patients may directly seek the services of a physical therapist without referral from a physician or other allied medical professionals. However, this call for independence requires ample degree of professional practice in dealing with clients or patients. The primary objective of the researchers in conducting this study is to determine the level of professional practice among physical therapists. This research utilized the descriptive-survey design to determine the level of professional practice among 40 physical therapists in Northern Mindanao (Region 10). Using convenience sampling, the respondents were the licensed physical therapists working as full-time staff among physical therapy facilities which catered patients/clients of varied clinical conditions. Almost all of the indicators for professional practice are "mostly" done by the physical therapists.
\end{abstract}


An enhancement of their professional practice skills is deemed necessary for them to consistently exhibit safety, conduct professionally, demonstrate accountability, communicate effectively, and culturally competent in every patient encounter.

Keywords: Professional practice, physical therapy, continuing professional education, professional development

\section{INTRODUCTION}

The clinical practice of physical therapy in the Philippines is moving towards full autonomy and direct access to patients. This development makes a physical therapist becomes a primary care provider and that patients may directly seek the services of a physical therapist without a referral from a physician or other allied medical professionals. However, this call for independence requires ample degree of professional practice in dealing with clients or patients.

With the involvement of physical therapists in primary care, it is important that the level of professional practice should be identified. Identification of the extent of professional practice will aid us in assessing and evaluating clinical competence which may be deemed necessary to promote consistent high standards of health care, delineate the present scope of physical therapy practice, and provide data that will guide the curricula of physical therapist professional, post-professional education and continuing professional development.

This theme has been a very common topic in the medical field research papers. However, for Physical Therapy in the Philippines there is limited research conducted regarding the professional practice, and none had been carried out in Northern Mindanao. The absence of such information and knowledge will put us behind the continuing progress in skills and technology from the rest of the world.

The primary objective of the researchers in conducting this study was to determine the extent of professional practice among physical therapists in Northern Mindanao.

\section{OBJECTIVES OF THE STUDY}

This study primarily sought to determine the extent of professional practice among physical therapists in Northern Mindanao. Specifically, this study aimed to: 1) determine the demographic characteristics of physical therapists in terms of gender, number of years in practice, highest degree awarded, employment, and direct patient service; and 2) assess the extent of professional practice among the physical therapists in terms of safety, professional behavior, accountability, communication, cultural competence, and professional development. 


\section{CONCEPTUAL FRAMEWORK}

Physical therapy is a dynamic profession with an established theoretical and scientific bases and widespread clinical applications in the restoration, maintenance, and promotion of optimal physical function.

As essential participants in the health care delivery system, physical therapists assume leadership roles in rehabilitation; in prevention, health maintenance, and programs that promote health, wellness, and fitness; and in professional and community organizations. Physical therapists also play important roles both in developing standards for physical therapist practice and in developing health care policy to ensure availability, accessibility, and optimal delivery of physical therapy services. The positive impact of physical therapists' services on health-related quality of life is well accepted (Guide to PT Practice, 2008).

The Philippine Physical Therapy Association (PPTA), 2014 elaborates that physical therapy is concerned with identifying and maximizing quality of life and movement potential within the spheres of promotion, prevention, treatment/intervention, habilitation and rehabilitation. This encompasses physical, psychological, emotional, and social well-being. Physical therapy involves the interaction between physical therapist, patients/clients, other health professionals, families, caregivers, and communities in a process where movement potential is assessed, and goals are agreed upon, using knowledge and skills unique to physical therapists.

In the Philippines, physical therapy is an established and regulated profession, with specific professional aspects of clinical practice and education, indicative of diversity in social, economic, cultural, and political contexts.

The idea of reflecting on professional practice now appears more explicit in physical therapy education. Physical therapy education always included both the accumulation of professional facts and practice understanding. Students become physical therapists by demonstrating professional knowledge by successfully completing the didactic component of the curricula, and passing a written board examination.

The safety of the patient/client, the success of the individual student, and the reputation of the physical therapy program all ride on students' abilities to apply the concepts that they learn in the classroom to real people in the real world (Cuffia, 2008). The physical therapist bears primary responsibility for the safety of each patient, regardless of treatment provided and, in some situations, who provides it (Fairchild, 2007).

Patient transfers, changes in position, exercise activities, and the transport equipment or patients have the potential to cause injury; therefore physical therapist must maintain a safe environment. Incidents leading to patient injuries can be linked to the use of improperly functioning or poorly maintained equipment, a physical 
setting with hazardous obstacles or congested space, an excessive number of patients in the treatment area in relation to the personnel available to treat them, and the limited availability of personnel (Pierson, 2007).

Physical therapists working in clinical settings must be aware of patient safety on a daily basis. Patient safety is a complex issue involving all members of the health care team. Because "raising awareness of the problem is fundamental to improving patient safety," physiotherapists need to be aware of all of the elements of patient safety on a daily basis, regardless of their role and work environment (Wong, 2004).

Accountability is an active acceptance by the physical therapist the responsibility for the diverse roles, obligations, and actions of the physical therapist, including selfregulation and other behaviors that positively influence patient/client outcomes, the profession, and the health needs of society (Guide to PT Practice- Core Values, 2003).

As stated by Fairchild (2007), communication among persons is a primary function of life. For the physical therapist, communication with patients, family members, other practitioners, and co-workers is a necessity. The physical therapist should recognized that different forms of communication such as verbal, nonverbal, and attentive listening, may be required depending on the purpose or situation related to the communication. According to Roberts (2013), communication in clinical encounters is vital in ensuring a positive experience and outcome for both patient and clinician.

Grignon et al. (2014) defined communication as the ability to exchange thoughts and information via verbal, nonverbal, and written language to enhance interactions and mutual understanding in varied situations, with people of diverse backgrounds. In the outcome for physical therapy education, communication is the ability of the students to interact/communicate with patients/clients, caregivers, health care providers, and community members in a manner that is congruent with situational and cultural needs.

Oral communication is a significant component of physical therapy service. The physical therapist must talk with a variety of individuals, including the patient, family members, referral sources, and additional practitioners providing care for the patient. Frequently a combination of oral and written communication is needed to provide a persuasive argument on behalf of the patient. The physical therapist must be skilled communicators to describe their course of action (Pagliarulo, 2012).

Communication also encompasses interviewing skills of the physical therapist. It requires careful nurturing and refinement over time. Even the most experienced healthcare professional should self-assess and work toward improvement. Adopting a compassionate and caring attitude, monitoring communication style, and being 
aware of cultural differences will help ensure successful communication (Goodman, 2007). Interviewing a patient/client and communicating effectively both verbally and non-verbally, with compassionate caring takes into consideration individual differences and the patient's/client's emotional and psychological needs (Normative Model of PT Professional Education, 2004).

The use of consistent language and the manner in which oral and written instructions or directions are given to a patient should enhance the patient's level of understanding and capacity to learn. This concept is particularly important when complex activities are being taught and when a patient's mental capacities have been altered (Pierson, 2007).

Cultural competence is a set of academic and interpersonal skills that allow individuals to increase their understanding and appreciation of cultural differences and similarities within, among, and between groups. This requires willingness and ability to draw on knowledgeable persons of and from the community in developing focused interventions, communications, and other supports (Pierson, 2002).

In preparation for patient care, the physical therapist should be aware of his or her own personal biases, prejudices, attitudes, and values to better understand the effect these beliefs may exert on a patient if they are applied injudiciously. The physical therapist should learn about or research the cultural norms and traditions associated with different ethnic or religious groups before treatment to be able to exhibit desirable behavior toward those individuals and their family members (Pierson, 2002).

According to the Normative Model of Physical Therapist Education, cultural competence is, "a set of congruent behaviors, attitudes, and policies that come together in a system, agency or among professionals that enables effective work in cross-cultural situations" (American Physical Therapy Association, 2000). Cross, Bazron, Dennis, and Isaacs (1989), further describe cultural competence as occurring on a continuum consisting of six positions, starting with cultural destructiveness on one end and cultural proficiency on the other. In comparison, Stam (1996), describes cultural competence as having five components: "(a) awareness and acceptance of cultural differences, (b) self-awareness, (c) understanding the dynamics of cultural differences, (d) knowledge of the client's family culture, (e) adaptation of services to support the client's culture."

Cultural competency establishes a foundation of mutual respect and understanding, which in turn allows patients and practitioners to communicate successfully with each other. Cultural and linguistic competence is a set of congruent behaviors, attitudes and policies that come together in a system, agency or among professionals that enables effective work in cross-cultural situations (US Department of Health and Human Services, 1999). Health care professionals must recognize 
the impact of culture on issues of health care access, service delivery models, and willingness to participate in care.

Cultural competence is a critical skill for health care providers to cultivate as regard for individual patient differences facilitates positive health outcomes (Bender, 2006). Effective care requires an understanding of the patient's values, family structure, and culture as a patient-provider interaction may be impeded when the respective parties are from divergent ethnic or cultural (cross-cultural) backgrounds.

The globalization of society, coupled with the burgeoning of study abroad opportunities, challenges educators of physical therapist students to design pedagogy that increases student exposure to diverse cultures and prepares them for rendering culturally competent care. While it is imperative to include opportunities for cultural exposure within physical therapy curricula, it is equally important to assess the resultant learning outcomes and impact on student development as professionals.

Grignon (2014) defined professional growth and development as advancing one's practice through continuous acquisition, refinement, and application of knowledge and skills. Examples of this outcome included being a lifelong learner, aspiring to and attaining advanced practice credentials, demonstrating continued competence, and engaging in reflective practice. Physical therapy schools are formulating plans for personal and professional development and lifelong learning based on self-reflective practice and feedback from others.

\section{METHODOLOGY}

This research utilized the descriptive-survey design to determine the level of professional practice among 40 physical therapists in Northern Mindanao (Region 10). Using convenience sampling, the respondents were the licensed physical therapists working as full-time staff among physical therapy facilities which catered patients/clients of varied clinical conditions.

The study used the Physical Therapist Clinical Performance Instrument of the American Physical Therapy Association (APTA), Department of Physical Therapy Education, 2006. The researchers added questions about the demographic characteristics of physical therapists. The researchers were directly responsible for the administration of the questionnaires. Demographic characteristics of the respondents were gathered using a query method which involved personal interview conducted by the researchers. Direct observations were done to rate the level of professional practice of the physical therapists. Demographic characteristics were determined using frequency and percentage. Computation of mean was done to determine level of professional practice of physical therapists. 


\section{RESULTS AND DISCUSSION}

Table 1 presents the demographic characteristics of physical therapists. Majority $(70 \%)$ of them are female while $30 \%$ are male. In terms of the number of years in practice, $45 \%$ are working as PT clinicians between $0-5$ years, $25 \%$ are working between $11-15$ years, $20 \%$ are working between 16 to 20 years, and gladly there is one (1) working for more than 20 years. The data denote that half of the physical therapists are working less than 10 years as clinicians because they are new graduates or newly licensed physical therapists and still on the process of mastering the clinical practice of the profession. Those working for more than ten years are assumed to be experts in the physical therapy professional practice.

Table 1. Demographic Characteristics of Physical Therapists

\begin{tabular}{|c|c|c|}
\hline Demographic Characteristics & Frequency & Percentage \\
\hline \multicolumn{3}{|l|}{ Gender } \\
\hline Male & 12 & 30 \\
\hline Female & 28 & 70 \\
\hline Total & 40 & 100 \\
\hline \multicolumn{3}{|l|}{ Number of Years In Practice } \\
\hline $0-5$ years & 18 & 45 \\
\hline $6-10$ years & 3 & 7.5 \\
\hline $11-15$ years & 10 & 25 \\
\hline $16-20$ years & 8 & 20 \\
\hline More than 20 years & 1 & 2.5 \\
\hline Total & 40 & 100 \\
\hline \multicolumn{3}{|l|}{ Highest Degree Awarded } \\
\hline Bachelor & 39 & 97.5 \\
\hline Master & 1 & 2.5 \\
\hline Doctorate & 0 & 0.0 \\
\hline Total & 40 & 100 \\
\hline \multicolumn{3}{|l|}{ Employment } \\
\hline General hospital & 11 & 27.5 \\
\hline Rehabilitation center & 27 & 67.5 \\
\hline Home health care & 0 & 0.0 \\
\hline Private practice & 2 & 5.0 \\
\hline Total & 40 & 100 \\
\hline \multicolumn{3}{|l|}{ Direct Patient Service } \\
\hline $0-10 \%$ & 5 & 12.5 \\
\hline $11-25 \%$ & 1 & 2.5 \\
\hline $26-50 \%$ & 4 & 10 \\
\hline $51-75 \%$ & 8 & 20 \\
\hline More than $75 \%$ & 22 & 55 \\
\hline Total & 40 & 100 \\
\hline
\end{tabular}

Almost all (97.5\%) of the physical therapists are bachelor's degree holder and only one (1) has a master's degree. The data suggest that the physical therapists are encouraged to pursue higher education or field of specialization for continuing professional development.

The majority (68\%) of the physical therapists are presently employed in the 
rehabilitation centers while $28 \%$ are connected in the general hospitals. Only two (2) or $5 \%$ of them are into private practice. Physical therapists who are working in the rehabilitation centers are more into specialized practice that cater patients with orthopedic, neurologic and pediatric conditions while those working in general hospitals have varied clinical experience because they usually cater diverse patient conditions.

More than half $(55 \%)$ of them have more than $75 \%$ direct patient service while $20 \%$ have $51-75 \%$ direct patient service. This denotes that when physical therapists have more direct patient service the more they will enhance their professional practice. Important to mention, that $15 \%$ of them have $0-25 \%$ direct patient service for the reason that they have a limited number of patients and relied much from physicians referral.

Table 2. Extent of Professional Practice of Physical Therapists on Safety

\begin{tabular}{lcc}
\hline \multicolumn{1}{c}{ Indicators } & Mean & Verbal Description \\
\hline $\begin{array}{l}\text { Establishes and maintains safe working environment. } \\
\text { Recognizes physiological and psychological changes in patients and adjusts }\end{array}$ & 3.50 & Always \\
patient interventions accordingly. & & Mostly \\
$\begin{array}{l}\text { Demonstrates awareness of contraindications and precautions of patient } \\
\text { intervention. }\end{array}$ & 3.23 & Mostly \\
$\begin{array}{l}\text { Ensures the safety of self, patient, and others throughout the clinical interaction } \\
\text { Requests assistance when necessary. }\end{array}$ & 3.38 & Mostly \\
Uses acceptable techniques for safe handling of patients & 3.26 & Mostly \\
Demonstrates knowledge of facility safety policies and procedures. & 3.41 & Mostly \\
\multicolumn{1}{c}{ Overall } & 3.43 & Mostly \\
\hline
\end{tabular}

Table 2 presents the extent of professional practice of physical therapist on safety. The data reveals that the physical therapists always (3.50) establish and maintain safe working environment as a very important protocol in all aspects of patient care. It is also imperative to mention that during clinical interactions, interns ensure the safety not only of self but most importantly the patient and others. Vital protocols prior to patient intervention are the recognition of the physiological and psychological changes of patients and awareness of contraindications and precautions that are developed and observed by interns during patient intervention. Such precautionary measures are given emphasis by Pierson (2007) and O'Sullivan (2014) to physical therapist during patient care.

However, almost all of the indicators for professional practice on safety are mostly done by the physical therapists such as the recognition of physiological and psychological changes in patients and adjusts patient intervention accordingly (3.08), demonstration of awareness of contraindications and precautions of patient intervention (3.23) and request assistance when necessary (3.26). The data imply 
that there is still a need to improve this area of professional practice to ensure safety during patient care. Fairchild (2007) emphasize that physical therapist bear the primary responsibility for safety of patient and should, therefore, maintain a safe environment Pierson (2007). Wong (2004) also added that physical therapist should be aware of all the elements of patient safety on a daily basis, regardless of their role and work environment. In fact, Scheirton (2007) concluded that acquiring knowledge of patient safety; learning new concepts, strategies and skills for preventing and reducing harm; making necessary practice changes; and, morally managing error all contribute to creating a culture of safety in physical therapy practice.

\section{Table 3. Extent of Professional Practice of Physical Therapists on Professional Behavior}

\begin{tabular}{lcc}
\hline \multicolumn{1}{c}{ Indicators } & Mean & Verbal Description \\
\hline Demonstrates initiative & 3.22 & Mostly \\
Is punctual and dependable. & 3.08 & Mostly \\
Wears attire consistent with expectations of the practice setting. & 3.60 & Always \\
Demonstrates integrity in all interactions. & 3.18 & Mostly \\
Exhibits caring, compassion, and empathy in providing services to patients. & 3.33 & Mostly \\
Maintains productive working relationships with patients, families, CI, and & 3.47 & Mostly \\
others. & 3.17 & Mostly \\
Demonstrates behaviors that contribute to a positive work environment. & 3.00 & Mostly \\
Accepts feedback without defensiveness. & 3.02 & Mostly \\
Manages conflict in constructive ways. & 3.61 & Always \\
Maintains patient privacy and modesty. & 3.55 & Always \\
Values the dignity of patients as individuals. & 3.29 & Mostly \\
Seeks feedback from clinical instructor related to clinical performance. & 3.22 & Mostly \\
Provides effective feedback to CI related to clinical/teaching mentoring. & & Mostly \\
\hline \multicolumn{1}{c}{ Overall } & 3.08 & . \\
\hline
\end{tabular}

Table 3 shows the extent of professional practice of physical therapists on professional behavior. The overall mean rating for professional behavior is 3.08 (mostly). It is important to note that physical therapists always maintain patient privacy (3.61), wear attire consistent with expectations of the practice setting (3.60), and value the dignity of patients as individuals (3.55) in every patient encounter. These aspects of professional behavior are very important protocols in interacting with people in the clinical setting.

However, a majority of the indicators for professional behavior are "mostly" done by physical therapists during patient care. This means that physical therapists must have to improve their professional behavior such as accepting feedback without defensiveness (3.00), punctuality and dependability (3.08), demonstration of behaviors that contribute to a positive work environment (3.17), and demonstration of integrity in all interactions (3.18) since these are very important ingredients in ensuring workplace professionalism. Tsoumas (2002) in her dissertation claimed that 
health care professional must work as a member of teams and within groups. Seeking opinions and suggestions from other team members is essential for the development of a comprehensive plan of care that will ultimately benefit the patient. Koenig et.al. (2003) added that professional behaviors indicate the physical therapists initiative, time management skills, ability to direct his or her learning, and interpersonal and organizational skills and may be viewed as a barometer of his/her ability to function in an ever-changing practice arena.

Table 4. Extent of Professional Practice of Physical Therapists on Accountability

\begin{tabular}{|c|c|c|}
\hline Indicators & Mean & Verbal Description \\
\hline Places patient's needs above self interests. & 3.11 & Mostly \\
\hline $\begin{array}{l}\text { Identifies, acknowledges, and accepts responsibility for actions and reports } \\
\text { errors. }\end{array}$ & 3.41 & Mostly \\
\hline Takes steps to remedy errors in a timely manner. & 3.36 & Mostly \\
\hline Abides by policies and procedures of the practice setting. & 3.46 & Mostly \\
\hline Maintains patient confidentiality. & 3.57 & Always \\
\hline $\begin{array}{l}\text { Adheres to legal practice standards including all laws and institutional } \\
\text { regulations related to patient care. }\end{array}$ & 3.27 & Mostly \\
\hline Identifies ethical or legal concems and initiates action to address the concems. & 3.21 & Mostly \\
\hline $\begin{array}{l}\text { Displays generosity as evidenced in the use of time and effort to meet patient } \\
\text { needs. }\end{array}$ & 3.41 & Mostly \\
\hline $\begin{array}{l}\text { Recognizes the need for physical therapy services to under-served and under- } \\
\text { represented populations. }\end{array}$ & 3.26 & Mostly \\
\hline $\begin{array}{l}\text { Strives to provide patient/client services that go beyond expected standards of } \\
\text { practice. }\end{array}$ & 3.03 & Mostly \\
\hline Overall & 3.31 & Mostly \\
\hline
\end{tabular}

Table 4 presents the level of professional practice on accountability. The overall mean rating for accountability is 3.31 (Mostly). The data show that physical therapists always maintain patient confidentiality (3.57) during patient care.

However, a majority of the indicators of accountability are "mostly" done by the physical therapists. With this result, there is a need for the physical therapists to strive to provide patient/client services that go beyond expected standards of practice (3.03), place patient's needs above self interests (3.11), identify ethical or legal concerns and initiates action to address the concerns (3.21), and recognize the need for physical therapy services to under-served and under-represented populations (3.26). The Guide to PT Practice- Core Values (2003) noted that part of the responsibility of the physical therapist is the acceptance of diverse roles, obligations, and actions including self-regulation and other behaviors that positively influence patient/client outcomes, the profession, and the health needs of society. According to APTA (2006), accountability primarily points at the moral and legal responsibility that a health care professional has for their actions. 
Table 5. Extent of Professional Practice of Physical Therapists on Communication

\begin{tabular}{|c|c|c|}
\hline Indicators & Mean & Verbal Description \\
\hline Communicates, verbally and nonverbally, in a professional and timely manner. & 3.36 & Mostly \\
\hline Initiates communication in difficult situations. & 3.07 & Mostly \\
\hline Selects the most appropriate person(s) with whom to communicate. & 3.12 & Mostly \\
\hline $\begin{array}{l}\text { Communicates respect for the roles and contributions of all participants in } \\
\text { patient care }\end{array}$ & 3.61 & Always \\
\hline $\begin{array}{l}\text { Listens actively and attentively to understand what is being communicated by } \\
\text { others. }\end{array}$ & 3.43 & Mostly \\
\hline $\begin{array}{l}\text { Demonstrates professionally and technically correct written and verbal } \\
\text { communication without jargon. }\end{array}$ & 3.35 & Mostly \\
\hline $\begin{array}{l}\text { Communicates using nonverbalmessages that are consistent with intended } \\
\text { message. }\end{array}$ & 3.00 & Mostly \\
\hline Engages in ongoing dialogue with professional peers or team members. & 2.78 & Mostly \\
\hline Interprets and responds to the nonverbal communication of others. & 3.15 & Mostly \\
\hline $\begin{array}{l}\text { Evaluates effectiveness of his/her communication and modifies communication } \\
\text { accordingly. }\end{array}$ & 3.22 & Mostly \\
\hline Seeks and responds to feedback from multiple sources in providing patient care. & 2.71 & Mostly \\
\hline Adjusts style of communication based on target audience. & 3.03 & Mostly \\
\hline Communicates with the patient using language the patient can understand. & 3.12 & Mostly \\
\hline Overall & 3.15 & Mostly \\
\hline
\end{tabular}

Table 5 presents the extent of professional practice of physical therapists on communication. The data reveal an overall mean rating of 3.15 (Mostly) on communication. It is noted that physical therapists communicate respect for the roles and contributions of all participants in patient care (3.61).

Conversely, a majority of the indicators for communication are "mostly" exhibited by the physical therapists. To improve their communication skills during patient care, there is a need for them to undergo communication skills retooling emphasizing on seeking and responding to feedback from multiple sources in providing patient care (2.71), engaging in dialogue with professional peers or team members (2.78), communicating using nonverbal messages that are consistent with intended message (3.00), and adjusting style of communication based on target audience (3.03). Goodman (2007) emphasized that monitoring communication style and being aware of cultural differences will help ensure successful communication. Davis (1998) also points out that physical therapists must interact with individuals who are not functioning at their best, and this requires careful attention to communication skills to be effective during patient interaction.

During actual patient encounters, they already develop verbal and non-verbal communication skills in a professional and timely manner without using medical jargons. According to Roberts (2013), communication in clinical encounters is vital in ensuring a positive experience and outcome for both patient and clinician. Interns also develop their communication skills to engage themselves in discussing their patient case with their clinical supervisors and co-interns or during case presentation. 
Pagliarulo (2012) emphasized that physical therapists as clinicians must be skilled communicators to describe their course of action during patient management.

\section{Table 6. Extent of Professional Practice of Physical Therapists on Cultural Competence}

\begin{tabular}{lcc}
\hline \multicolumn{1}{c}{ Indicators } & Mean & Verbal Description \\
\hline $\begin{array}{l}\text { Incorporates an understanding of the implications of individual and cultural } \\
\text { differences and a dapts behavior accordingly in all aspects of physical therapy } \\
\text { services. }\end{array}$ & 3.33 & Mostly \\
$\begin{array}{l}\text { Communicates with sensitivity by considering differences in race/ethnicity, } \\
\text { religion, gender, age, national origin, sexual orientation, and disability or health } \\
\text { status }\end{array}$ & 3.35 & Mostly \\
$\begin{array}{l}\text { Provides care in a nonjudgmental manner when the patients' beliefs and values } \\
\text { conflict with the individual's belief system }\end{array}$ & 3.36 & Mostly \\
$\begin{array}{l}\text { Discovers, respects, and highly regard individual differences, preferences, } \\
\text { values, life issues, and emotional needs within and among cultures }\end{array}$ & 3.40 & Mostly \\
$\begin{array}{l}\text { Values the socio-cultural, psychological, and economic influences on patients } \\
\text { and clients and responds accordingly } \\
\text { Is aware of and suspends own social and cultural biases. }\end{array}$ & 3.36 & Mostly \\
$\quad$ Overall & 3.35 & Mostly \\
\hline
\end{tabular}

Table 6 presents the extent of professional practice of physical therapists on cultural competence. The data reveal that all indicators are "mostly" performed by the physical therapists with an overall mean rating of 3.36. This implies that the physical therapist must have to improve their level of cultural competence since the majority of their patients/clients come from a different socio-cultural background. As indicated by Bender (2006) that cultural competence is a critical skill for health care providers to cultivate as regard for individual patient differences facilitates positive health outcomes. Significantly, they must have to develop within them the sense of cultural awareness by suspending their personal social and cultural biases so as not to affect the outcomes of their patient management. Higgs and Hunt (1999) stressed that these skills did not come from knowledge alone, but that one must be aware of one's values first. Pierson (2002) also noted that in preparation for patient care, the physical therapist should be aware of his or her own personal biases, prejudices, attitudes, and values to better understand the effect these beliefs may exert on a patient if they are applied injudiciously. Interns also acquired skills that in their plan of care they considered the preferences, values, life issues, emotional needs, sociocultural, psychological, and economic influences of patients toward physical therapy intervention.

Moreover, during actual handling of patients, physical therapists must conversed with cultural sensitivity and provide care in a nonjudgmental manner considering differences in ethnicity, religion, gender, age, sexual orientation, and disability or 
health status of their patients. The physical therapist should learn about or research the cultural norms and traditions associated with different ethnic or religious groups before treatment to be able to exhibit desirable behavior toward those individuals and their family members Pierson (2002).

Table 7. Extent of Professional Practice of Physical Therapists on Professional Development

\begin{tabular}{|c|c|c|}
\hline Indicators & Mean & Verbal Description \\
\hline Identifies strengths and limitations in clinical performance & 2.47 & Occasionally \\
\hline Seeks guidance as necessary to address limitations & 2.58 & Mostly \\
\hline $\begin{array}{l}\text { Uses self-evaluation, ongoing feedback from others, inquiry, and reflection to } \\
\text { conduct regular ongoing self-assessment to improve clinical practice and } \\
\text { professional development }\end{array}$ & 2.31 & Occasionally \\
\hline $\begin{array}{l}\text { Acknowledges and accepts responsibility for and consequences of his or her } \\
\text { actions }\end{array}$ & 2.62 & Mostly \\
\hline $\begin{array}{l}\text { Establishes realistic short and long-term goals in a plan for professional } \\
\text { development }\end{array}$ & 2.61 & Mostly \\
\hline $\begin{array}{l}\text { Seeks out additional leaming experiences to enhance clinical and professional } \\
\text { performance }\end{array}$ & 2.53 & Mostly \\
\hline Discusses progress of clinical and professional growth & 2.30 & Occasionally \\
\hline Accepts responsibility for continuous professional leaming & 2.37 & Occasionally \\
\hline Discusses professional issues related to physical therapy practice & 2.37 & Occasionally \\
\hline Participates in professional activities beyond the practice environment & 2.00 & Occasionally \\
\hline $\begin{array}{l}\text { Provides to and receives feedback from peers regarding performance, behaviors, } \\
\text { and goals }\end{array}$ & 2.51 & Mostly \\
\hline $\begin{array}{l}\text { Provides current knowledge and theory (in-service, case presentation, joumal } \\
\text { club, projects, systematic data collection, etc) to achieve optimal patient care. }\end{array}$ & 2.00 & Occasionally \\
\hline Overall & 2.39 & Occasionally \\
\hline
\end{tabular}

Table 7 presents the extent of professional practice of physical therapists on professional development. The overall mean rating for professional development is 2.39 (Occasionally). Physical therapists mostly acknowledge and accept responsibility for and consequences of his or her actions (2.62) and establish realistic short and long-term goals in a plan for professional development (2.61). However, they occasionally provide current knowledge and theory to achieve optimal patient care (2.00), participate in professional activities beyond the practice environment (2.00), discuss progress of clinical and professional growth (2.30), use self-evaluation, ongoing feedback from others, inquiry, and reflection to conduct regular ongoing selfassessment to improve clinical practice and professional development (2.31), accept responsibility for continuous professional learning (2.37), discuss professional issues related to physical therapy practice (2.37), and identify strengths and limitations in clinical performance (2.47). This means that physical therapists must have to imbibe the importance of professional development in advancing themselves to practice the physical therapy profession in the future.

Today's physical therapy practitioner functions on a more autonomous level in the 
delivery of patient care which places a higher demand for professional development on the new graduates of the physical therapy educational programs (www.marquette. edu/physical-therapy/documents/ProfessionalBehaviors, 2010).

Grignon et.al. (2014) emphasized that joining professional activities such as seminar, workshops, symposium, trainings, etc. to discuss professional issues and learn new trends in physical therapy practice, seeking out additional learning experiences beyond the practice environment, and accepting responsibility for continuous professional learning are very important ingredients for professional growth and development. Schreiber (2009) added that the physical therapy profession has embraced the concept of evidence-based practice. The call for a commitment to evidence-based practice (EBP) in physical therapy has become more strident because providing evidence-based service to patients is considered a professional, moral and ethical obligation Ross (2004), Schreiber (2005), WCPT (2012).

May et al. (1995) and Sprafka (1999) emphasized that the ability to seek, use, and provide feedback and constructive criticism are essentials to improve interactions with others and health care professional must work as a member of teams and within groups. Seeking opinions and suggestions from other team members is essential for the development of comprehensive care plans.

\section{CONCLUSIONS}

Based on the findings of the study, it is concluded that the indicators for the professional practice of physical therapists are mostly demonstrated based on the standards of physical therapy clinical practice. An enhancement of their professional practice skills is deemed necessary for them to consistently exhibit safety, conduct professionally, demonstrate accountability, communicate effectively, and culturally competent in every patient encounter.

\section{LITERATURE CITED}

A Normative model of Physical Therapist Professional Education. 2004 American Physical Therapy Association: Alexandria, VA.

American Physical Therapy Association: Guide to Physical Therapist Practice. 2003 Revised Edition 2. Alexandria, VA: APTA.

American Physical Therapy Association: Guide to Physical Therapist Practice 2001 Physical Therapy. 81:9-744. 
Bender, D.G.

2006 Culture and Critical Care/Developing Cultural Competence in Physical Therapy Practice. Journal of Physical Therapy Education.

Commission on Higher Education Memorandum Order No. 24 Series 2006 2006 policies, standards and guidelines for physical therapy and occupational therapy education. www.ched.gov.ph/index.php /archive/cmo-archives/2006-ched-memorandum-orders-

Cross, T.L., Bazron, B.J., Dennis, K.W., \& Isaacs, M.R.

1989 Towards a culturally competent system of care: A monograph on effective services for minority children who are severely emotionally disturbed (vol. 1). Washington, DC: Georgetown University, National Technical Assistance Center for Children's Mental Health.

Cuffia, B.

2008 Physical Therapy Professional Foundations: Keys to Success in School and Career. Journal of Physical Therapy Education.

Goodman, C., Snyder, T.

2009 Differential Diagnosis for Physical Therapists: Screening for Referral. (4th Ed.). Elsevier: Singapore.

Grignon, T., Henley, E. Lee, K., Abentroth, M. Jette, D. 2014 Expected Graduate Outcomes in US Physical Therapist Education Programs: A Qualitative Study. Journal of Physical Therapy Education.

Guccione, A.

1991 Physical therapy diagnosis and the relationship between impairment and function. Physical Therapy. 71:449.

Herbert, R., Jamtvedt, G., Mead, J., Hagen, K.B.

2005 Practical evidence-based physiotherapy. London: Elsevier Butterworth Heinemann. 
Higgs, J., Jones, M.A.

2000 Clinical reasoning in the health professions. In: Higgs J, Jones ME, editors. Clinical reasoning in the health professions. (2nd Ed.). Boston, USA: Butterworth-Heinemann.

National Standards and an Outcomes-Focused Research Agenda.

1999 Office of Minority Health, Public Health Service, US Department of Health and Human Services.

O'Sullivan, S.B., Schmitz, T.J.

2014 Physical Rehabilitation: Assessment and Treatment. (5th Ed.). F.A. Davis Company: Philadelphia.

Pagliarulo, M.

2012 Introduction to Physical Therapy. (4th Ed.). Elsevier Mosby: Singapore.

Pierson, F.M., Fairchild, S.L.

2002 Principles and Techniques of Patient Care. (3rd Ed.).Elsevier: Singapore.

Professionalism in Physical Therapy: Core Values

2004 Normative Model.

Republic Act No. 5680

an Act Creating the Board of Examiners for Physical Therapists and Occupational Therapists. www.prc.gov.ph/prb/

Roberts, L. Whittle, C.

2013 Measuring Verbal Communication in Initial Physical Therapy Encounters. Journal of Physical Therapy.

Ross, E.C., Anderson, E.Z.

2004 The evolution of a physical therapy research curriculum: Integrating evidence-based practice and clinical decision making. Journal of Physical Therapy Education. 18:52-57.

Schreiber, J., Stern, P.

2005 A review of the literature on evidence-based practice in physical therapy. Internet J Allied Health Scientific Practice. 3:1-10. 
Schreiber, J., Downey, P.

2009 Academic Program Support for Evidence-Based Practice: A MixedMethods Investigation. Journal of Physical Therapy Education.

Stam, J.R.

1996 Family culture and chronic conditions.In Jackson P.L., Yessey J.A., (Eds).Primary care of the child with a chronic condition. St. Louis, MO: Mosby.

Wong, J., Beglaryan, H.

2004 Strategies for hospitals to improve patient safety: a review of the research. Toronto: The Change Foundation.

World Confederation for Physical Therapy policy statement on description of physical therapy wcpt.org/policy/ps-description PT.

World Confederation for Physical Therapy, Policy Statement on Evidence-Based Practice, Approved at the 15th General Meeting of WCPT June 2003 Retrieved: August 7, 2014. 